\title{
Diophantine Sets. Part II
}

\author{
Karol Pąk \\ Institute of Informatics \\ University of Białystok \\ Poland
}

Summary. The article is the next in a series aiming to formalize the MDPR-theorem using the Mizar proof assistant [3, 6], 4]. We analyze four equations from the Diophantine standpoint that are crucial in the bounded quantifier theorem, that is used in one of the approaches to solve the problem.

Based on our previous work 1, we prove that the value of a given binomial coefficient and factorial can be determined by its arguments in a Diophantine way. Then we prove that two products

$$
z=\prod_{i=1}^{x}(1+i \cdot y), \quad z=\prod_{i=1}^{x}(y+1-j),
$$

where $y>x$ are Diophantine.

The formalization follows [10, Z. Adamowicz, P. Zbierski 2] as well as M. Davis [5].

MSC: 11D45 68T99 03B35

Keywords: Hilbert's 10th problem; Diophantine relations

MML identifier: HILB10_4, version: 8.1.09 5.57.1355

\section{Product of Zero Based Finite Sequences}

From now on $i, j, n, n_{1}, n_{2}, m, k, l, u$ denote natural numbers, $r, r_{1}, r_{2}$ denote real numbers, $x, y$ denote integers, $a, b$ denote non trivial natural numbers, $F$ denotes a finite 0 -sequence, $\mathcal{F}, \mathcal{F}_{1}, \mathcal{F}_{2}$ denote complex-valued finite 0 -sequences, and $c, c_{1}, c_{2}$ denote complex numbers.

Let us consider $c_{1}$ and $c_{2}$. Let us note that $\left\langle c_{1}, c_{2}\right\rangle$ is complex-valued. 
Let $\mathcal{F}$ be a finite 0 -sequence. The functor $\prod \mathcal{F}$ yielding an element of $\mathbb{C}$ is defined by the term

(Def. 1) $\cdot \mathbb{C} \odot \mathcal{F}$.

Now we state the propositions:

(1) If $\mathcal{F}$ is real-valued, then $\prod \mathcal{F}=\cdot \mathbb{R} \odot \mathcal{F}$.

(2) If $\mathcal{F}$ is $\mathbb{Z}$ valued, then $\prod \mathcal{F}=\cdot \mathbb{Z} \odot \mathcal{F}$.

(3) If $\mathcal{F}$ is natural-valued, then $\prod \mathcal{F}=\cdot_{\mathbb{N}} \odot \mathcal{F}$.

Let $F$ be a real-valued finite 0 -sequence. One can check that $\prod F$ is real.

Let $F$ be a natural-valued finite 0 -sequence. One can verify that $\prod F$ is natural.

Now we state the propositions:

(4) If $\mathcal{F}=\emptyset$, then $\prod \mathcal{F}=1$.

(5) $\Pi\langle c\rangle=c$.

(6) $\prod\left\langle c_{1}, c_{2}\right\rangle=c_{1} \cdot c_{2}$

(7) $\prod\left(\mathcal{F}_{1} \frown \mathcal{F}_{2}\right)=\left(\prod \mathcal{F}_{1}\right) \cdot\left(\prod \mathcal{F}_{2}\right)$.

(8) $c+\mathcal{F}_{1} \frown \mathcal{F}_{2}=\left(c+\mathcal{F}_{1}\right) \frown\left(c+\mathcal{F}_{2}\right)$.

PRoOF: For every object $x$ such that $x \in \operatorname{dom}\left(c+\mathcal{F}_{1} \frown \mathcal{F}_{2}\right)$ holds $\left(c+\mathcal{F}_{1}\right\urcorner$ $\left.\mathcal{F}_{2}\right)(x)=\left(\left(c+\mathcal{F}_{1}\right) \frown\left(c+\mathcal{F}_{2}\right)\right)(x)$.

(9) $c_{1}+\left\langle c_{2}\right\rangle=\left\langle c_{1}+c_{2}\right\rangle$.

(10) Let us consider finite 0 -sequences $f_{1}, f_{2}$, and $n$. Suppose $n \leqslant \operatorname{len} f_{1}$. Then $\left(f_{1} \frown f_{2}\right)_{\lfloor n}=f_{1\lfloor n} \frown f_{2}$.

Let us consider $n$. One can verify that there exists a finite 0 -sequence which is $n$-element and natural-valued and there exists a finite 0 -sequence which is natural-valued and positive yielding.

Let $R$ be a positive yielding binary relation and $X$ be a set. Observe that $R \uparrow X$ is positive yielding.

Let $X$ be a positive yielding, real-valued finite 0 -sequence. One can verify that $\prod X$ is positive.

Now we state the proposition:

(11) Let us consider a natural-valued, positive yielding finite 0 -sequence $X$. If $i \in \operatorname{dom} X$, then $X(i) \leqslant \prod X$.

Proof: Define $\mathcal{P}$ [natural number] $\equiv$ for every natural-valued, positive yielding finite 0 -sequence $X$ for every natural number $i$ such that len $X=$ $\$_{1}$ and $i \in \operatorname{dom} X$ holds $X(i) \leqslant \prod X$. If $\mathcal{P}[n]$, then $\mathcal{P}[n+1]$. $\mathcal{P}[n]$.

Let $X$ be a natural-valued finite 0 -sequence and $n$ be a positive natural number. Let us observe that $n+X$ is positive yielding.

Now we state the proposition: 
(12) Let us consider natural-valued finite 0-sequences $X_{1}, X_{2}$. Suppose len $X_{1}$ $=\operatorname{len} X_{2}$ and for every $n$ such that $n \in \operatorname{dom} X_{1}$ holds $X_{1}(n) \leqslant X_{2}(n)$. Then $\prod X_{1} \leqslant \prod X_{2}$.

Proof: Define $\mathcal{P}$ [natural number] $\equiv$ for every natural-valued finite 0sequences $X_{1}, X_{2}$ such that len $X_{1}=\$_{1}=$ len $X_{2}$ and for every $n$ such that $n \in \operatorname{dom} X_{1}$ holds $X_{1}(n) \leqslant X_{2}(n)$ holds $\prod X_{1} \leqslant \prod X_{2} . \mathcal{P}[0]$. If $\mathcal{P}[n]$, then $\mathcal{P}[n+1] . \mathcal{P}[n]$.

\section{Binomial is Diophantine}

Now we state the propositions:

(13) If $k \leqslant n$, then $\left(\begin{array}{l}n \\ k\end{array}\right) \leqslant n^{k}$.

Proof: Define $\mathcal{P}$ [natural number] $\equiv$ if $\$_{1} \leqslant n$, then $\left(\begin{array}{c}n \\ \$_{1}\end{array}\right) \leqslant n^{\$_{1}}$. If $\mathcal{P}[m]$, then $\mathcal{P}[m+1] . \mathcal{P}[m]$.

(14) If $u>n^{k}$ and $n \geqslant k>i$, then $\left(\begin{array}{c}n \\ i\end{array}\right) \cdot\left(u^{i}\right)<\frac{u^{k}}{n}$. The theorem is a consequence of (13).

(15) If $u>n^{k}$ and $n \geqslant k$, then $\left\lfloor\frac{(u+1)^{n}}{u^{k}}\right\rfloor \bmod u=\left(\begin{array}{l}n \\ k\end{array}\right)$.

Proof: Set $I=\left\langle\left(\begin{array}{l}n \\ 0\end{array}\right) 1^{0} u^{n}, \ldots,\left(\begin{array}{l}n \\ n\end{array}\right) 1^{n} u^{0}\right\rangle$. Set $k_{1}=k+1$. Consider $q$ being a finite sequence such that $I=\left(I\left\lceil k_{1}\right) \frown q\right.$. Reconsider $I_{1}=I$ as a finite sequence of elements of $\mathbb{N}$. Set $k_{2}=k \mapsto \frac{u^{k}}{n}$. For every natural number $i$ such that $i \in \operatorname{Seg} k$ holds $\left(I_{1}\lceil k)(i)<k_{2}(i)\right.$. Define $\mathcal{P}$ [natural number, object $] \equiv \$_{2} \in \mathbb{N}$ and for every natural number $i$ such that $i=\$_{2}$ holds $q\left(\$_{1}\right)=u^{k} \cdot u \cdot i$. For every natural number $j$ such that $j \in \operatorname{Seg} \operatorname{len} q$ there exists an object $x$ such that $\mathcal{P}[j, x]$. Consider $Q$ being a finite sequence such that $\operatorname{dom} Q=\operatorname{Seg} \operatorname{len} q$ and for every natural number $j$ such that $j \in \operatorname{Seg}$ len $q$ holds $\mathcal{P}[j, Q(j)]$. rng $Q \subseteq \mathbb{N}$. For every natural number $i$ such that $1 \leqslant i \leqslant \operatorname{len} q$ holds $q(i)=\left(u^{k} \cdot u \cdot Q\right)(i) .\left\lfloor\frac{\sum I_{1}}{u^{k}}\right\rfloor=\left(\begin{array}{l}n \\ k\end{array}\right)+u \cdot\left(\sum Q\right)$. $\left(\begin{array}{l}n \\ k\end{array}\right) \leqslant n^{k}$.

(16) Let us consider natural numbers $x, y, z$. Then $x \geqslant z$ and $y=\left(\begin{array}{l}x \\ z\end{array}\right)$ if and only if there exist natural numbers $u, v, y_{1}, y_{2}, y_{3}$ such that $y_{1}=x^{z}$ and $y_{2}=(u+1)^{x}$ and $y_{3}=u^{z}$ and $u>y_{1}$ and $v=\left\lfloor\frac{y_{2}}{y_{3}}\right\rfloor$ and $y \equiv v(\bmod u)$ and $y<u$ and $x \geqslant z$.

PROOF: If $x \geqslant z$ and $y=\left(\begin{array}{l}x \\ z\end{array}\right)$, then there exist natural numbers $u, v, y_{1}$, $y_{2}, y_{3}$ such that $y_{1}=x^{z}$ and $y_{2}=(u+1)^{x}$ and $y_{3}=u^{z}$ and $u>y_{1}$ and $v=\left\lfloor\frac{y_{2}}{y_{3}}\right\rfloor$ and $y \equiv v(\bmod u)$ and $y<u$ and $x \geqslant z \cdot y \bmod u=\left(\begin{array}{l}x \\ z\end{array}\right)$. 


\section{Factorial is Diophantine}

Now we state the propositions:

(17) If $k>0$ and $n>2 \cdot k^{k+1}$, then $k !=\left\lfloor\frac{n^{k}}{\left(\begin{array}{c}n \\ k\end{array}\right)}\right\rfloor$.

(18) Let us consider natural numbers $x, y$. Then $y=x$ ! if and only if there exist natural numbers $n, y_{1}, y_{2}, y_{3}$ such that $y_{1}=2 \cdot x^{x+1}$ and $y_{2}=n^{x}$ and $y_{3}=\left(\begin{array}{l}n \\ x\end{array}\right)$ and $n>y_{1}$ and $y=\left\lfloor\frac{y_{2}}{y_{3}}\right\rfloor$.

PRoOF: If $y=x$ !, then there exist natural numbers $n, y_{1}, y_{2}, y_{3}$ such that $y_{1}=2 \cdot x^{x+1}$ and $y_{2}=n^{x}$ and $y_{3}=\left(\begin{array}{l}n \\ x\end{array}\right)$ and $n>y_{1}$ and $y=\left\lfloor\frac{y_{2}}{y_{3}}\right\rfloor$.

\section{Diophanticity of Selected Products}

In the sequel $x, y, x_{1}, u, w$ denote natural numbers.

Now we state the propositions:

(19) Let us consider natural numbers $x_{1}, w, u$. Suppose $x_{1} \cdot w \equiv 1(\bmod u)$. Let us consider a natural number $x$. Then $\prod\left(1+x_{1} \cdot(\operatorname{idseq}(x))\right) \equiv x_{1}{ }^{x}$. $(x !) \cdot\left(\begin{array}{c}w+x \\ x\end{array}\right)(\bmod u)$.

Proof: Consider $b$ being an integer such that $u \cdot b=x_{1} \cdot w-1$. Define $\mathcal{P}$ [natural number $] \equiv \prod\left(1+x_{1} \cdot\left(\operatorname{idseq}\left(\$_{1}\right)\right)\right) \equiv x_{1}{ }^{\$_{1}} \cdot\left(\$_{1} !\right) \cdot\left(\begin{array}{c}w+\$_{1} \\ \$_{1}\end{array}\right)(\bmod u)$. If $\mathcal{P}[n]$, then $\mathcal{P}[n+1]$ by [12, (43)]. $\mathcal{P}[n]$.

(20) Let us consider natural numbers $x, y, x_{1}$. Suppose $x_{1} \geqslant 1$. Then $y=$ $\prod\left(1+x_{1} \cdot(\operatorname{idseq}(x))\right)$ if and only if there exist natural numbers $u, w, y_{1}$, $y_{2}, y_{3}, y_{4}, y_{5}$ such that $u>y$ and $x_{1} \cdot w \equiv 1(\bmod u)$ and $y_{1}=x_{1}{ }^{x}$ and $y_{2}=x !$ and $y_{3}=\left(\begin{array}{c}w+x \\ x\end{array}\right)$ and $y_{1} \cdot y_{2} \cdot y_{3} \equiv y(\bmod u)$ and $y_{4}=1+x_{1} \cdot x$ and $y_{5}=y_{4}{ }^{x}$ and $u>y_{5}$.

Proof: Define $\mathcal{P}$ [natural number $] \equiv\left(1+x_{1} \cdot \$_{1}\right)^{\$_{1}} \geqslant \prod\left(1+x_{1} \cdot\left(\right.\right.$ idseq $\left.\left.\left(\$_{1}\right)\right)\right)$. If $\mathcal{P}[n]$, then $\mathcal{P}[n+1] . \mathcal{P}[n]$. If $y=\prod\left(1+x_{1} \cdot(\operatorname{idseq}(x))\right)$, then there exist natural numbers $u, w, y_{1}, y_{2}, y_{3}, y_{4}, y_{5}$ such that $u>y$ and $x_{1} \cdot w \equiv 1(\bmod u)$ and $y_{1}=x_{1}{ }^{x}$ and $y_{2}=x !$ and $y_{3}=\left(\begin{array}{c}w+x \\ x\end{array}\right)$ and $y_{1} \cdot y_{2} \cdot y_{3} \equiv y(\bmod u)$ and $y_{4}=1+x_{1} \cdot x$ and $y_{5}=y_{4}{ }^{x}$ and $u>y_{5}$ by $\left[8\right.$, (16)]. Set $U=x_{1}^{x} \cdot(x !) \cdot\left(\begin{array}{c}w+x \\ x\end{array}\right)$. $\prod\left(1+x_{1} \cdot(\operatorname{idseq}(x))\right) \equiv U(\bmod u)$.

(21) $\quad c_{1}+n \mapsto c_{2}=n \mapsto\left(c_{1}+c_{2}\right)$.

(22) Let us consider natural numbers $x, y, x_{1}$. If $x_{1}=0$, then $y=\prod\left(1+x_{1}\right.$. (idseq $(x)))$ iff $y=1$. The theorem is a consequence of (21).

(23) If $n \geqslant k$, then $\prod(n+1+-\operatorname{idseq}(k))=k ! \cdot\left(\begin{array}{l}n \\ k\end{array}\right)$.

Proof: Define $\mathcal{P}$ [natural number] $\equiv$ if $\$_{1} \leqslant n$, then $\Pi\left(n+1+-\right.$ idseq $\left.\left(\$_{1}\right)\right)$ $=\$_{1} ! \cdot\left(\begin{array}{c}n \\ \$_{1}\end{array}\right)$. If $\mathcal{P}[i]$, then $\mathcal{P}[i+1]$ by [7, (3), (2)]. $\mathcal{P}[i]$. 
(24) Let us consider natural numbers $y, x_{1}, x_{2}$. Then $y=\prod\left(x_{2}+1+\right.$ -idseq $\left.\left(x_{1}\right)\right)$ and $x_{2}>x_{1}$ if and only if $y=x_{1} ! \cdot\left(\begin{array}{l}x_{2} \\ x_{1}\end{array}\right)$ and $x_{2}>x_{1}$.

\section{Selected Subsets of Zero Based Finite Sequences of $\mathbb{N}$ as Diophantine Sets}

From now on $n, m, k$ denote natural numbers, $p, q$ denote $n$-element finite 0 -sequences of $\mathbb{N}, i_{1}, i_{2}, i_{3}, i_{4}, i_{5}, i_{6}$ denote elements of $n$, and $a, b, d, f$ denote integers.

Now we state the propositions:

(25) Let us consider natural numbers $a, b, i_{1}, i_{2}$, and $i_{3}$. Then $\left\{p: p\left(i_{1}\right)=\right.$ $\left.\left(a \cdot p\left(i_{2}\right)+b\right) \cdot p\left(i_{3}\right)\right\}$ is a Diophantine subset of the $n$-xtuples of $\mathbb{N}$.

Proof: Define $\mathcal{R}$ (natural number, natural number, natural number) $=a$. $\$_{1}+b$. Define $\mathcal{P}_{1}$ [natural number, natural number, natural object, natural number, natural number, natural number $] \equiv 1 \cdot \$_{1}=1 \cdot \$_{3} \cdot \$_{2}$. For every $n, i_{1}$, $i_{2}, i_{3}, i_{4}$, and $i_{5},\left\{p: \mathcal{P}_{1}\left[p\left(i_{1}\right), p\left(i_{2}\right), \mathcal{R}\left(p\left(i_{3}\right), p\left(i_{4}\right), p\left(i_{5}\right)\right), p\left(i_{3}\right), p\left(i_{4}\right), p\left(i_{5}\right)\right]\right\}$ is a Diophantine subset of the $n$-xtuples of $\mathbb{N}$. Define $\mathcal{Q}_{1}$ [finite 0 -sequence of $\mathbb{N}] \equiv \mathcal{P}_{1}\left[\$_{1}\left(i_{1}\right), \$_{1}\left(i_{3}\right), a \cdot \$_{1}\left(i_{2}\right)+b, \$_{1}\left(i_{3}\right), \$_{1}\left(i_{3}\right), \$_{1}\left(i_{3}\right)\right]$. Define $\mathcal{Q}_{2}[$ finite 0 -sequence of $\mathbb{N}] \equiv \$_{1}\left(i_{1}\right)=\left(a \cdot \$_{1}\left(i_{2}\right)+b\right) \cdot \$_{1}\left(i_{3}\right) .\left\{p: \mathcal{Q}_{1}[p]\right\}=\left\{q: \mathcal{Q}_{2}[q]\right\}$.

(26) $\left\{p: p\left(i_{1}\right)=a \cdot p\left(i_{2}\right) \cdot p\left(i_{3}\right)\right\}$ is a Diophantine subset of the $n$-xtuples of $\mathbb{N}$.

Proof: Define $\mathcal{Q}_{1}[$ finite 0 -sequence of $\mathbb{N}] \equiv 1 \cdot \$_{1}\left(i_{1}\right)=a \cdot \$_{1}\left(i_{2}\right) \cdot \$_{1}\left(i_{3}\right)$. Define $\mathcal{Q}_{2}[$ finite 0 -sequence of $\mathbb{N}] \equiv \$_{1}\left(i_{1}\right)=a \cdot \$_{1}\left(i_{2}\right) \cdot \$_{1}\left(i_{3}\right) \cdot\left\{p: \mathcal{Q}_{1}[p]\right\}=$ $\left\{q: \mathcal{Q}_{2}[q]\right\}$.

(27) Let us consider a Diophantine subset $A$ of the $n$-xtuples of $\mathbb{N}$, and natural numbers $k, n_{4}$. Suppose $k+n_{4}=n$. Then $\left\{p_{\left\lfloor n_{4}\right.}: p \in A\right\}$ is a Diophantine subset of the $k$-xtuples of $\mathbb{N}$.

Proof: Consider $n_{3}$ being a natural number, $p_{1}$ being a $\mathbb{Z}$ valued polynomial of $n+n_{3}, \mathbb{R}_{F}$ such that for every object $s, s \in A$ iff there exists an $n$-element finite 0 -sequence $x$ of $\mathbb{N}$ and there exists an $n_{3}$-element finite 0 -sequence $y$ of $\mathbb{N}$ such that $s=x$ and $\operatorname{eval}\left(p_{1},{ }^{\circledR}\left(x^{\frown} y\right)\right)=0$. Reconsider $I=\mathrm{id}_{n+n_{3}}$ as a finite 0-sequence. Set $I_{1}=I\left\lceil n_{4}\right.$. Set $I_{2}=\left(I\lceil n)_{\left\lfloor n_{4}\right.}\right.$. Set $I_{3}=I_{\lfloor n}$. Reconsider $J=\left(I_{2} \frown I_{1}\right)^{\frown} I_{3}$ as a function from $n+n_{3}$ into $n+n_{3}$. Set $h=$ the $p_{1}$ permuted by $J^{-1}$. Reconsider $H=h$ as a polynomial of $k+\left(n_{4}+n_{3}\right), \mathbb{R}_{\mathrm{F}}$. Set $Y=\left\{p_{\left\lfloor n_{4}\right.}: p \in A\right\}$. $Y \subseteq$ the $k$-xtuples of $\mathbb{N}$. For every object $s, s \in Y$ iff there exists a $k$-element finite 0 -sequence $x$ of $\mathbb{N}$ and there exists an $\left(n_{4}+n_{3}\right)$-element finite 0 -sequence $y$ of $\mathbb{N}$ such that $s=x$ and $\operatorname{eval}\left(H,{ }^{@}(x \frown y)\right)=0$ by $[9,(25)],[11,(27)]$. 
(28) Let us consider integers $a, b$, a natural number $c, i_{1}, i_{2}$, and $i_{3}$. Then $\left\{p: a \cdot p\left(i_{1}\right)=\left\lfloor\frac{b \cdot p\left(i_{2}\right)}{c \cdot p\left(i_{3}\right)}\right\rfloor\right.$ and $\left.c \cdot p\left(i_{3}\right) \neq 0\right\}$ is a Diophantine subset of the $n$-xtuples of $\mathbb{N}$.

Proof: Define $\mathcal{F}_{2}$ (natural number, natural number, natural number) $=$ $c \cdot \$_{3}+a \cdot c \cdot \$_{1} \cdot \$_{3}$. For every $n, i_{1}, i_{2}, i_{3}, i_{4}$, and $d,\left\{p: \mathcal{F}_{2}\left(p\left(i_{1}\right), p\left(i_{2}\right), p\left(i_{3}\right)\right)=\right.$ $\left.d \cdot p\left(i_{4}\right)\right\}$ is a Diophantine subset of the $n$-xtuples of $\mathbb{N}$. Define $\mathcal{P}_{2}$ [natural number, natural number, integer $] \equiv b \cdot \$_{1}+0<\$_{3}$. For every $n, i_{1}, i_{2}$, $i_{3}, i_{4}$, and $i_{5},\left\{p: \mathcal{P}_{2}\left[p\left(i_{1}\right), p\left(i_{2}\right), \mathcal{F}_{2}\left(p\left(i_{3}\right), p\left(i_{4}\right), p\left(i_{5}\right)\right)\right]\right\}$ is a Diophantine subset of the $n$-xtuples of $\mathbb{N}$. Define $\mathcal{P}_{3}$ [natural number, natural number, integer $] \equiv b \cdot \$_{1} \geqslant \$_{3}+0$. Define $\mathcal{F}_{3}$ (natural number, natural number, natural number $)=a \cdot c \cdot \$_{1} \cdot \$_{3}$. For every $n, i_{1}, i_{2}, i_{3}, i_{4}$, and $i_{5},\left\{p: \mathcal{P}_{3}\left[p\left(i_{1}\right), p\left(i_{2}\right), \mathcal{F}_{3}\left(p\left(i_{3}\right), p\left(i_{4}\right), p\left(i_{5}\right)\right)\right]\right\}$ is a Diophantine subset of the $n$-xtuples of $\mathbb{N}$.

Define $\mathcal{Q}_{1}[$ finite 0 -sequence of $\mathbb{N}] \equiv \mathcal{P}_{2}\left[\$_{1}\left(i_{2}\right), \$_{1}\left(i_{2}\right), \mathcal{F}_{2}\left(\$_{1}\left(i_{1}\right), \$_{1}\left(i_{1}\right)\right.\right.$, $\left.\$_{1}\left(i_{3}\right)\right)$ ]. Define $\mathcal{Q}_{2}[$ finite 0 -sequence of $\mathbb{N}] \equiv \mathcal{P}_{3}\left[\$_{1}\left(i_{2}\right), \$_{1}\left(i_{2}\right), \mathcal{F}_{3}\left(\$_{1}\left(i_{1}\right)\right.\right.$, $\left.\left.\$_{1}\left(i_{1}\right), \$_{1}\left(i_{3}\right)\right)\right]$. Define $\mathcal{Q}_{12}$ [finite 0 -sequence of $\left.\mathbb{N}\right] \equiv \mathcal{Q}_{1}\left[\$_{1}\right]$ and $\mathcal{Q}_{2}\left[\$_{1}\right]$. Define $\mathcal{Q}_{3}[$ finite 0 -sequence of $\mathbb{N}] \equiv c \cdot \$_{1}\left(i_{3}\right) \neq 0 \cdot \$_{1}\left(i_{3}\right)+0$. Define $\mathcal{Q}_{123}$ [finite 0 -sequence of $\mathbb{N}] \equiv \mathcal{Q}_{12}\left[\$_{1}\right]$ and $\mathcal{Q}_{3}\left[\$_{1}\right]$. Define $\mathcal{T}$ [finite 0 -sequence of $\left.\mathbb{N}\right] \equiv$ $a \cdot \$_{1}\left(i_{1}\right)=\left\lfloor\frac{b \cdot \$_{1}\left(i_{2}\right)}{c \cdot \$_{1}\left(i_{3}\right)}\right\rfloor$ and $c \cdot \$_{1}\left(i_{3}\right) \neq 0 .\left\{p: \mathcal{Q}_{1}[p]\right.$ and $\left.\mathcal{Q}_{2}[p]\right\}$ is a Diophantine subset of the $n$-xtuples of $\mathbb{N}$. $\left\{p: \mathcal{Q}_{12}[p]\right.$ and $\left.\mathcal{Q}_{3}[p]\right\}$ is a Diophantine subset of the $n$-xtuples of $\mathbb{N}$. For every $p, \mathcal{T}[p]$ iff $\mathcal{Q}_{123}[p] .\{p: \mathcal{T}[p]\}=\{q$ : $\left.\mathcal{Q}_{123}[q]\right\}$.

Let us consider $i_{1}, i_{2}$, and $i_{3}$. Now we state the propositions:

(29) If $n \neq 0$, then $\left\{p: p\left(i_{1}\right) \geqslant p\left(i_{3}\right)\right.$ and $\left.p\left(i_{2}\right)=\left(\begin{array}{l}p\left(i_{1}\right) \\ p\left(i_{3}\right)\end{array}\right)\right\}$ is a Diophantine subset of the $n$-xtuples of $\mathbb{N}$.

Proof: Set $n_{6}=n+6$. Define $\mathcal{R}[$ finite 0 -sequence of $\mathbb{N}] \equiv \$_{1}\left(i_{1}\right) \geqslant \$_{1}\left(i_{3}\right)$ and $\$_{1}\left(i_{2}\right)=\left(\begin{array}{c}\$_{1}\left(i_{1}\right) \\ \$_{1}\left(i_{3}\right)\end{array}\right)$. Set $R R=\{p: \mathcal{R}[p]\}$. Reconsider $X=i_{1}, Y=i_{2}$, $Z=i_{3}, U=n, V=n+1, Y_{1}=n+2, Y_{2}=n+3, Y_{3}=n+4, U_{1}=n+5$ as an element of $n+6$. Define $\mathcal{P}_{1}$ [finite 0 -sequence of $\mathbb{N}$ ] $\equiv \$_{1}\left(Y_{1}\right)=$ $\$_{1}(X)^{\$_{1}(Z)}$. Define $\mathcal{P}_{2}[$ finite 0 -sequence of $\mathbb{N}] \equiv \$_{1}\left(Y_{2}\right)=\$_{1}\left(U_{1}\right)^{\$_{1}(X)}$. Define $\mathcal{P}_{3}$ [finite 0 -sequence of $\left.\mathbb{N}\right] \equiv \$_{1}\left(Y_{3}\right)=\$_{1}(U)^{\$_{1}(Z)}$. Define $\mathcal{P}_{4}$ [finite 0 -sequence of $\mathbb{N}] \equiv 1 \cdot \$_{1}(U)>1 \cdot \$_{1}\left(Y_{1}\right)+0$. Define $\mathcal{P}_{5}$ [finite 0 -sequence of $\mathbb{N}] \equiv 1 \cdot \$_{1}(V)=\left\lfloor\frac{1 \cdot \$_{1}\left(Y_{2}\right)}{1 \cdot \$_{1}\left(Y_{3}\right)}\right\rfloor$ and $1 \cdot \$_{1}\left(Y_{3}\right) \neq 0 .\left\{p\right.$, where $p$ is an $n_{6}$-element finite 0 -sequence of $\left.\mathbb{N}: \mathcal{P}_{5}[p]\right\}$ is a Diophantine subset of the $n_{6}$-xtuples of $\mathbb{N}$.

Define $\mathcal{P}_{6}[$ finite 0 -sequence of $\mathbb{N}] \equiv 1 \cdot \$_{1}(Y) \equiv 1 \cdot \$_{1}(V)\left(\bmod 1 \cdot \$_{1}(U)\right)$. Define $\mathcal{P}_{7}$ [finite 0 -sequence of $\left.\mathbb{N}\right] \equiv 1 \cdot \$_{1}(U)>1 \cdot \$_{1}(Y)+0$. Define $\mathcal{P}_{8}$ [finite 0 -sequence of $\mathbb{N}$ ] $\equiv 1 \cdot \$_{1}(X) \geqslant 1 \cdot \$_{1}(Z)+0$. Define $\mathcal{P}_{9}$ [finite 0 -sequence of $\mathbb{N}] \equiv 1 \cdot \$_{1}\left(U_{1}\right)=1 \cdot \$_{1}(U)+1$. Define $\mathcal{P}_{12}$ [finite 0 -sequence of $\left.\mathbb{N}\right] \equiv$ 
$\mathcal{P}_{1}\left[\$_{1}\right]$ and $\mathcal{P}_{2}\left[\$_{1}\right] .\left\{p\right.$, where $p$ is an $n_{6}$-element finite 0 -sequence of $\mathbb{N}$ : $\left.\mathcal{P}_{12}[p]\right\}$ is a Diophantine subset of the $n_{6}$-xtuples of $\mathbb{N}$. Define $\mathcal{P}_{123}$ [finite 0 -sequence of $\mathbb{N}] \equiv \mathcal{P}_{12}\left[\$_{1}\right]$ and $\mathcal{P}_{3}\left[\$_{1}\right] .\left\{p\right.$, where $p$ is an $n_{6}$-element finite 0 -sequence of $\left.\mathbb{N}: \mathcal{P}_{123}[p]\right\}$ is a Diophantine subset of the $n_{6}$-xtuples of $\mathbb{N}$. Define $\mathcal{P}_{1234}[$ finite 0 -sequence of $\mathbb{N}] \equiv \mathcal{P}_{123}\left[\$_{1}\right]$ and $\mathcal{P}_{4}\left[\$_{1}\right] .\{p$, where $p$ is an $n_{6}$-element finite 0 -sequence of $\left.\mathbb{N}: \mathcal{P}_{1234}[p]\right\}$ is a Diophantine subset of the $n_{6}$-xtuples of $\mathbb{N}$. Define $\mathcal{P}_{12345}[$ finite 0 -sequence of $\mathbb{N}] \equiv \mathcal{P}_{1234}\left[\$_{1}\right]$ and $\mathcal{P}_{5}\left[\$_{1}\right] .\left\{p\right.$, where $p$ is an $n_{6}$-element finite 0 -sequence of $\left.\mathbb{N}: \mathcal{P}_{12345}[p]\right\}$ is a Diophantine subset of the $n_{6}$-xtuples of $\mathbb{N}$.

Define $\mathcal{P}_{123456}[$ finite 0 -sequence of $\mathbb{N}] \equiv \mathcal{P}_{12345}\left[\$_{1}\right]$ and $\mathcal{P}_{6}\left[\$_{1}\right] .\{p$, where $p$ is an $n_{6}$-element finite 0 -sequence of $\left.\mathbb{N}: \mathcal{P}_{123456}[p]\right\}$ is a Diophantine subset of the $n_{6}$-xtuples of $\mathbb{N}$. Define $\mathcal{P}_{1234567}$ [finite 0 -sequence of $\mathbb{N}$ ] $\equiv$ $\mathcal{P}_{123456}\left[\$_{1}\right]$ and $\mathcal{P}_{7}\left[\$_{1}\right] .\left\{p\right.$, where $p$ is an $n_{6}$-element finite 0 -sequence of $\left.\mathbb{N}: \mathcal{P}_{1234567}[p]\right\}$ is a Diophantine subset of the $n_{6}$-xtuples of $\mathbb{N}$. Define $\mathcal{P}_{12345678}[$ [inite 0 -sequence of $\mathbb{N}] \equiv \mathcal{P}_{1234567}\left[\$_{1}\right]$ and $\mathcal{P}_{8}\left[\$_{1}\right] .\{p$, where $p$ is an $n_{6}$-element finite 0 -sequence of $\left.\mathbb{N}: \mathcal{P}_{12345678}[p]\right\}$ is a Diophantine subset of the $n_{6}$-xtuples of $\mathbb{N}$. Define $\mathcal{P}_{123456789}[$ finite 0 -sequence of $\mathbb{N}$ ] $\equiv$ $\mathcal{P}_{12345678}\left[\$_{1}\right]$ and $\mathcal{P}_{9}\left[\$_{1}\right]$. Set $P P=\left\{p\right.$, where $p$ is an $n_{6}$-element finite 0-sequence of $\left.\mathbb{N}: \mathcal{P}_{123456789}[p]\right\} . P P$ is a Diophantine subset of the $n_{6^{-}}$ xtuples of $\mathbb{N}$. Reconsider $P P_{n}=\left\{p\left\lceil n\right.\right.$, where $p$ is an $n_{6}$-element finite 0 -sequence of $\mathbb{N}: p \in P P\}$ as a Diophantine subset of the $n$-xtuples of $\mathbb{N}$. $P P_{n} \subseteq R R . R R \subseteq P P_{n}$.

(30) $\left\{p: p\left(i_{1}\right) \geqslant p\left(i_{3}\right)\right.$ and $\left.p\left(i_{2}\right)=\left(\begin{array}{l}p\left(i_{1}\right) \\ p\left(i_{3}\right)\end{array}\right)\right\}$ is a Diophantine subset of the $n$ xtuples of $\mathbb{N}$. The theorem is a consequence of (29).

Let us consider $i_{1}$ and $i_{2}$. Now we state the propositions:

(31) If $n \neq 0$, then $\left\{p: p\left(i_{1}\right)=p\left(i_{2}\right)\right.$ ! $\}$ is a Diophantine subset of the $n$-xtuples of $\mathbb{N}$.

Proof: Set $n_{6}=n+6$. Define $\mathcal{R}[$ finite 0 -sequence of $\mathbb{N}] \equiv \$_{1}\left(i_{1}\right)=\$_{1}\left(i_{2}\right)$ !. Set $R R=\{p: \mathcal{R}[p]\}$. Reconsider $Y=i_{1}, X=i_{2}, N=n, Y_{1}=n+1$, $Y_{2}=n+2, Y_{3}=n+3, X_{1}=n+4, X_{2}=n+5$ as an element of $n+6$. Define $\mathcal{P}_{1}$ [finite 0-sequence of $\left.\mathbb{N}\right] \equiv \$_{1}\left(Y_{1}\right)=\$_{1}\left(X_{2}\right)^{\$_{1}\left(X_{1}\right)}$. Define $\mathcal{P}_{2}$ [finite 0 -sequence of $\mathbb{N}] \equiv \$_{1}\left(Y_{2}\right)=\$_{1}(N)^{\$_{1}(X)}$. Define $\mathcal{P}_{3}$ [finite 0-sequence of $\mathbb{N}] \equiv \$_{1}(N) \geqslant \$_{1}(X)$ and $\$_{1}\left(Y_{3}\right)=\left(\begin{array}{l}\$_{1}(N) \\ \$_{1}(X)\end{array}\right)$. $\left\{p\right.$, where $p$ is an $n_{6}$-element finite 0 -sequence of $\left.\mathbb{N}: \mathcal{P}_{3}[p]\right\}$ is a Diophantine subset of the $n_{6}$-xtuples of $\mathbb{N}$. Define $\mathcal{P}_{4}[$ finite 0 -sequence of $\mathbb{N}] \equiv 1 \cdot \$_{1}(Y)=\left\lfloor\frac{1 \cdot \$_{1}\left(Y_{2}\right)}{1 \cdot \$_{1}\left(Y_{3}\right)}\right\rfloor$ and 1 . $\$_{1}\left(Y_{3}\right) \neq 0$. $\left\{p\right.$, where $p$ is an $n_{6}$-element finite 0 -sequence of $\left.\mathbb{N}: \mathcal{P}_{4}[p]\right\}$ is a Diophantine subset of the $n_{6}$-xtuples of $\mathbb{N}$. Define $\mathcal{P}_{5}$ [finite 0 -sequence of $\mathbb{N}] \equiv 1 \cdot \$_{1}\left(X_{2}\right)=2 \cdot \$_{1}(X)+0$. Define $\mathcal{P}_{6}$ [finite 0 -sequence of $\left.\mathbb{N}\right] \equiv$ $1 \cdot \$_{1}\left(X_{1}\right)=1 \cdot \$_{1}(X)+1$. Define $\mathcal{P}_{7}[$ finite 0 -sequence of $\mathbb{N}] \equiv 1 \cdot \$_{1}(N)>$ 
$1 \cdot \$_{1}\left(Y_{1}\right)+0$. Define $\mathcal{P}_{12}[$ finite 0 -sequence of $\mathbb{N}] \equiv \mathcal{P}_{1}\left[\$_{1}\right]$ and $\mathcal{P}_{2}\left[\$_{1}\right] .\{p$ where $p$ is an $n_{6}$-element finite 0 -sequence of $\left.\mathbb{N}: \mathcal{P}_{12}[p]\right\}$ is a Diophantine subset of the $n_{6}$-xtuples of $\mathbb{N}$.

Define $\mathcal{P}_{123}$ [finite 0 -sequence of $\left.\mathbb{N}\right] \equiv \mathcal{P}_{12}\left[\$_{1}\right]$ and $\mathcal{P}_{3}\left[\$_{1}\right]$. $\{p$, where $p$ is an $n_{6}$-element finite 0 -sequence of $\left.\mathbb{N}: \mathcal{P}_{123}[p]\right\}$ is a Diophantine subset of the $n_{6}$-xtuples of $\mathbb{N}$. Define $\mathcal{P}_{1234}$ [finite 0-sequence of $\left.\mathbb{N}\right] \equiv \mathcal{P}_{123}\left[\$_{1}\right.$ ] and $\mathcal{P}_{4}\left[\$_{1}\right] .\left\{p\right.$, where $p$ is an $n_{6}$-element finite 0 -sequence of $\left.\mathbb{N}: \mathcal{P}_{1234}[p]\right\}$ is a Diophantine subset of the $n_{6}$-xtuples of $\mathbb{N}$. Define $\mathcal{P}_{12345}$ [finite 0 -sequence of $\mathbb{N}] \equiv \mathcal{P}_{1234}\left[\$_{1}\right]$ and $\mathcal{P}_{5}\left[\$_{1}\right]$. $\left\{p\right.$, where $p$ is an $n_{6}$-element finite 0 -sequence of $\left.\mathbb{N}: \mathcal{P}_{12345}[p]\right\}$ is a Diophantine subset of the $n_{6}$-xtuples of $\mathbb{N}$. Define $\mathcal{P}_{123456}[$ finite 0 -sequence of $\mathbb{N}] \equiv \mathcal{P}_{12345}\left[\$_{1}\right]$ and $\mathcal{P}_{6}\left[\$_{1}\right]$. $\{p$, where $p$ is an $n_{6}$-element finite 0 -sequence of $\left.\mathbb{N}: \mathcal{P}_{123456}[p]\right\}$ is a Diophantine subset of the $n_{6}$-xtuples of $\mathbb{N}$. Define $\mathcal{P}_{1234567}$ [finite 0 -sequence of $\left.\mathbb{N}\right] \equiv \mathcal{P}_{123456}\left[\$_{1}\right]$ and $\mathcal{P}_{7}\left[\$_{1}\right]$. Set $P P=\left\{p\right.$, where $p$ is an $n_{6}$-element finite 0 -sequence of $\left.\mathbb{N}: \mathcal{P}_{1234567}[p]\right\} . P P$ is a Diophantine subset of the $n_{6}$-xtuples of $\mathbb{N}$. Reconsider $P P_{n}=\left\{p\left\lceil n\right.\right.$, where $p$ is an $n_{6}$-element finite 0 -sequence of $\mathbb{N}: p \in P P\}$ as a Diophantine subset of the $n$-xtuples of $\mathbb{N} . P P_{n} \subseteq R R$. $R R \subseteq P P_{n}$.

(32) $\left\{p: p\left(i_{1}\right)=p\left(i_{2}\right) !\right\}$ is a Diophantine subset of the $n$-xtuples of $\mathbb{N}$. The theorem is a consequence of (31).

(33) $\left\{p: 1+\left(p\left(i_{1}\right)+1\right) \cdot\left(p\left(i_{2}\right) !\right)=p\left(i_{3}\right)\right\}$ is a Diophantine subset of the $n$ xtuples of $\mathbb{N}$.

Proof: Define $\mathcal{R}$ (natural number, natural number, natural number) $=1$. $\$_{1}+-1$. Define $\mathcal{P}_{1}$ [natural number, natural number, integer] $\equiv 1 \cdot \$_{1} \cdot \$_{2}=$ $\$_{3}$. For every $i_{1}, i_{2}, i_{3}, i_{4}$, and $i_{5},\left\{p: \mathcal{P}_{1}\left[p\left(i_{1}\right), p\left(i_{2}\right), \mathcal{R}\left(p\left(i_{3}\right), p\left(i_{4}\right), p\left(i_{5}\right)\right)\right]\right\}$ is a Diophantine subset of the $n$-xtuples of $\mathbb{N}$. Define $\mathcal{F}_{2}$ (natural number, natural number, natural number $)=\$ 1$ !. For every $i_{1}, i_{2}, i_{3}$, and $i_{4},\{p$ $\left.: \mathcal{F}_{2}\left(p\left(i_{1}\right), p\left(i_{2}\right), p\left(i_{3}\right)\right)=p\left(i_{4}\right)\right\}$ is a Diophantine subset of the $n$-xtuples of $\mathbb{N}$. Define $\mathcal{P}_{2}$ [natural number, natural number, natural object, natural number, natural number, natural number $] \equiv 1 \cdot \$_{1} \cdot \$_{3}=1 \cdot \$_{2}-1$.

For every $i_{1}, i_{2}, i_{3}, i_{4}$, and $i_{5},\left\{p: \mathcal{P}_{2}\left[p\left(i_{1}\right), p\left(i_{2}\right), \mathcal{F}_{2}\left(p\left(i_{3}\right), p\left(i_{4}\right), p\left(i_{5}\right)\right)\right.\right.$, $\left.\left.p\left(i_{3}\right), p\left(i_{4}\right), p\left(i_{5}\right)\right]\right\}$ is a Diophantine subset of the $n$-xtuples of $\mathbb{N}$. Define $\mathcal{P}_{3}$ [natural number, natural number, natural object, natural number, natural number, natural number $] \equiv 1 \cdot \$_{3} \cdot\left(\$_{1} !\right)=1 \cdot \$_{2}-1$. Define $\mathcal{F}_{3}$ (natural number, natural number, natural number $)=1 \cdot \$_{1}+1$. For every $n, i_{1}, i_{2}$, $i_{3}, i_{4}$, and $i_{5},\left\{p: \mathcal{P}_{3}\left[p\left(i_{1}\right), p\left(i_{2}\right), \mathcal{F}_{3}\left(p\left(i_{3}\right), p\left(i_{4}\right), p\left(i_{5}\right)\right), p\left(i_{3}\right), p\left(i_{4}\right), p\left(i_{5}\right)\right]\right\}$ is a Diophantine subset of the $n$-xtuples of $\mathbb{N}$. Define $\mathcal{Q}_{1}$ [finite 0 -sequence of $\mathbb{N}] \equiv \mathcal{P}_{3}\left[\$_{1}\left(i_{2}\right), \$_{1}\left(i_{3}\right), 1 \cdot \$_{1}\left(i_{1}\right)+1, \$_{1}\left(i_{3}\right), \$_{1}\left(i_{3}\right), \$_{1}\left(i_{3}\right)\right]$. Define $\mathcal{Q}_{2}$ [finite 0 -sequence of $\mathbb{N}] \equiv 1+\left(\$_{1}\left(i_{1}\right)+1\right) \cdot\left(\$_{1}\left(i_{2}\right) !\right)=\$_{1}\left(i_{3}\right) \cdot\left\{p: \mathcal{Q}_{1}[p]\right\}=\{q$ $\left.: \mathcal{Q}_{2}[q]\right\}$. 
Let us consider $i_{1}, i_{2}$, and $i_{3}$. Now we state the propositions:

(34) If $n \neq 0$, then $\left\{p: p\left(i_{3}\right)=\prod\left(1+p\left(i_{1}\right) \cdot\left(\operatorname{idseq}\left(p\left(i_{2}\right)\right)\right)\right)\right.$ and $\left.p\left(i_{1}\right) \geqslant 1\right\}$ is a Diophantine subset of the $n$-xtuples of $\mathbb{N}$.

Proof: Set $n_{12}=n+13$. Define $\mathcal{R}$ [finite 0 -sequence of $\left.\mathbb{N}\right] \equiv \$_{1}\left(i_{3}\right)=\prod(1+$ $\left.\$_{1}\left(i_{1}\right) \cdot\left(\operatorname{idseq}\left(\$_{1}\left(i_{2}\right)\right)\right)\right)$ and $\$_{1}\left(i_{1}\right) \geqslant 1$. Set $R R=\{p: \mathcal{R}[p]\}$. Reconsider $X_{1}=i_{1}, X=i_{2}, Y=i_{3}, U=n, W=n+1, Y_{1}=n+2, Y_{2}=n+3$, $Y_{3}=n+4, Y_{4}=n+5, Y_{5}=n+6, X_{3}=n+7, W_{1}=n+8, Y_{6}=n+9$, $Y_{7}=n+10, X_{4}=n+11, O=n+12$ as an element of $n_{12}$. Define $\mathcal{Q}$ [finite 0 -sequence of $\mathbb{N}] \equiv 1 \cdot \$_{1}\left(X_{1}\right) \geqslant 0 \cdot \$_{1}(Y)+1$. Define $\mathcal{P}_{1}[$ finite 0-sequence of $\mathbb{N}$ ] $\equiv 1 \cdot \$_{1}(U)>1 \cdot \$_{1}(Y)+0$. Define $\mathcal{P}_{2}$ [finite 0-sequence of $\mathbb{N}] \equiv 1 \cdot \$_{1}\left(X_{3}\right)=1 \cdot \$_{1}\left(X_{1}\right) \cdot \$_{1}(W)$.

Define $\mathcal{P}_{3}[$ finite 0 -sequence of $\mathbb{N}] \equiv \$_{1}(O)=1$. Define $\mathcal{P}_{4}[$ finite 0 -sequence of $\mathbb{N}] \equiv 1 \cdot \$_{1}\left(X_{3}\right) \equiv 1 \cdot \$_{1}(O)\left(\bmod 1 \cdot \$_{1}(U)\right)$. Define $\mathcal{P}_{5}$ [finite 0-sequence of $\mathbb{N}] \equiv \$_{1}\left(Y_{1}\right)=\$_{1}\left(X_{1}\right)^{\$_{1}(X)}$. Define $\mathcal{P}_{6}$ [finite 0-sequence of $\mathbb{N}] \equiv \$_{1}\left(Y_{2}\right)=\$_{1}(X)$ !. $\left\{p\right.$, where $p$ is an $n_{12}$-element finite 0 -sequence of $\left.\mathbb{N}: \mathcal{P}_{6}[p]\right\}$ is a Diophantine subset of the $n_{12}$-xtuples of $\mathbb{N}$. Define $\mathcal{P}_{7}[$ finite 0 -sequence of $\mathbb{N}] \equiv 1 \cdot \$_{1}\left(W_{1}\right)=1 \cdot \$_{1}(W)+1 \cdot \$_{1}(X)+0$. Define $\mathcal{P}_{8}$ [finite 0 -sequence of $\left.\mathbb{N}\right] \equiv \$_{1}\left(W_{1}\right) \geqslant \$_{1}(X)$ and $\$_{1}\left(Y_{3}\right)=\left(\begin{array}{c}\$_{1}\left(W_{1}\right) \\ \$_{1}(X)\end{array}\right)$. $\left\{p\right.$, where $p$ is an $n_{12}$-element finite 0 -sequence of $\left.\mathbb{N}: \mathcal{P}_{8}[p]\right\}$ is a Diophantine subset of the $n_{12}$-xtuples of $\mathbb{N}$. Define $\mathcal{P}_{9}$ [finite 0 -sequence of $\mathbb{N}] \equiv 1 \cdot \$_{1}\left(Y_{6}\right)=1 \cdot \$_{1}\left(Y_{1}\right) \cdot \$_{1}\left(Y_{2}\right)$. Define $\mathcal{P} \mathcal{A}[$ finite 0 -sequence of $\mathbb{N}] \equiv 1 \cdot \$_{1}\left(Y_{7}\right)=1 \cdot \$_{1}\left(Y_{6}\right) \cdot \$_{1}\left(Y_{3}\right)$. Define $\mathcal{P B}$ [inite 0 -sequence of $\left.\mathbb{N}\right] \equiv$ $1 \cdot \$_{1}\left(Y_{7}\right) \equiv 1 \cdot \$_{1}(Y)\left(\bmod 1 \cdot \$_{1}(U)\right)$. Define $\mathcal{P C}$ [finite 0 -sequence of $\mathbb{N}] \equiv 1 \cdot \$_{1}\left(X_{4}\right)=1 \cdot \$_{1}\left(X_{1}\right) \cdot \$_{1}(X)$. Define $\mathcal{P D}$ [finite 0 -sequence of $\left.\mathbb{N}\right] \equiv$ $1 \cdot \$_{1}\left(Y_{4}\right)=1 \cdot \$_{1}\left(X_{4}\right)+1$. Define $\mathcal{P E}[$ finite 0 -sequence of $\mathbb{N}] \equiv \$_{1}\left(Y_{5}\right)=$ $\$_{1}\left(Y_{4}\right)^{\$_{1}(X)}$. Define $\mathcal{P F}$ [finite 0-sequence of $\left.\mathbb{N}\right] \equiv 1 \cdot \$_{1}(U)>1 \cdot \$_{1}\left(Y_{5}\right)+0$.

Define $\mathcal{C}_{1}$ [finite 0 -sequence of $\left.\mathbb{N}\right] \equiv \mathcal{Q}\left[\$_{1}\right]$ and $\mathcal{P}_{1}\left[\$_{1}\right] .\{p$, where $p$ is an $n_{12}$-element finite 0 -sequence of $\left.\mathbb{N}: \mathcal{C}_{1}[p]\right\}$ is a Diophantine subset of the $n_{12}$-xtuples of $\mathbb{N}$. Define $\mathcal{C}_{2}$ [finite 0 -sequence of $\left.\mathbb{N}\right] \equiv \mathcal{C}_{1}\left[\$_{1}\right]$ and $\mathcal{P}_{2}\left[\$_{1}\right]$. $\left\{p\right.$, where $p$ is an $n_{12}$-element finite 0 -sequence of $\left.\mathbb{N}: \mathcal{C}_{2}[p]\right\}$ is a Diophantine subset of the $n_{12}$-xtuples of $\mathbb{N}$. Define $\mathcal{C}_{3}$ [finite 0 -sequence of $\left.\mathbb{N}\right] \equiv \mathcal{C}_{2}\left[\$_{1}\right.$ ] and $\mathcal{P}_{3}\left[\$_{1}\right] .\left\{p\right.$, where $p$ is an $n_{12}$-element finite 0 -sequence of $\left.\mathbb{N}: \mathcal{C}_{3}[p]\right\}$ is a Diophantine subset of the $n_{12}$-xtuples of $\mathbb{N}$. Define $\mathcal{C}_{4}$ [finite 0 -sequence of $\mathbb{N}] \equiv \mathcal{C}_{3}\left[\$_{1}\right]$ and $\mathcal{P}_{4}\left[\$_{1}\right] .\left\{p\right.$, where $p$ is an $n_{12}$-element finite 0 -sequence of $\left.\mathbb{N}: \mathcal{C}_{4}[p]\right\}$ is a Diophantine subset of the $n_{12}$-xtuples of $\mathbb{N}$. Define $\mathcal{C}_{5}[$ finite 0 -sequence of $\mathbb{N}] \equiv \mathcal{C}_{4}\left[\$_{1}\right]$ and $\mathcal{P}_{5}\left[\$_{1}\right] .\left\{p\right.$, where $p$ is an $n_{12}$-element finite 0 -sequence of $\left.\mathbb{N}: \mathcal{C}_{5}[p]\right\}$ is a Diophantine subset of the $n_{12}$-xtuples of $\mathbb{N}$. Define $\mathcal{C}_{6}$ [finite 0 -sequence of $\left.\mathbb{N}\right] \equiv \mathcal{C}_{5}\left[\$_{1}\right]$ and $\mathcal{P}_{6}\left[\$_{1}\right] .\left\{p\right.$, where $p$ is an $n_{12^{-}}$ element finite 0 -sequence of $\left.\mathbb{N}: \mathcal{C}_{6}[p]\right\}$ is a Diophantine subset of the $n_{12}$ xtuples of $\mathbb{N}$. Define $\mathcal{C}_{7}$ [finite 0 -sequence of $\left.\mathbb{N}\right] \equiv \mathcal{C}_{6}\left[\$_{1}\right]$ and $\mathcal{P}_{7}\left[\$_{1}\right]$. $\{p$, 
where $p$ is an $n_{12}$-element finite 0 -sequence of $\left.\mathbb{N}: \mathcal{C}_{7}[p]\right\}$ is a Diophantine subset of the $n_{12}$-xtuples of $\mathbb{N}$. Define $\mathcal{C}_{8}$ [finite 0 -sequence of $\left.\mathbb{N}\right] \equiv \mathcal{C}_{7}\left[\$_{1}\right]$ and $\mathcal{P}_{8}\left[\$_{1}\right] .\left\{p\right.$, where $p$ is an $n_{12}$-element finite 0 -sequence of $\left.\mathbb{N}: \mathcal{C}_{8}[p]\right\}$ is a Diophantine subset of the $n_{12}$-xtuples of $\mathbb{N}$. Define $\mathcal{C}_{9}$ [finite 0 -sequence of $\mathbb{N}] \equiv \mathcal{C}_{8}\left[\$_{1}\right]$ and $\mathcal{P}_{9}\left[\$_{1}\right]$. \{ $\left\{p\right.$, where $p$ is an $n_{12}$-element finite 0 -sequence of $\left.\mathbb{N}: \mathcal{C}_{9}[p]\right\}$ is a Diophantine subset of the $n_{12}$-xtuples of $\mathbb{N}$.

Define $\mathcal{C} \mathcal{A}$ [finite 0 -sequence of $\mathbb{N}] \equiv \mathcal{C}_{9}\left[\$_{1}\right]$ and $\mathcal{P} \mathcal{A}\left[\$_{1}\right]$. \{ $\{$, where $p$ is an $n_{12}$-element finite 0 -sequence of $\left.\mathbb{N}: \mathcal{C A}[p]\right\}$ is a Diophantine subset of the $n_{12}$-xtuples of $\mathbb{N}$. Define $\mathcal{C B}$ [finite 0 -sequence of $\left.\mathbb{N}\right] \equiv \mathcal{C A}\left[\$_{1}\right]$ and $\mathcal{P B}\left[\$_{1}\right] .\left\{p\right.$, where $p$ is an $n_{12}$-element finite 0 -sequence of $\left.\mathbb{N}: \mathcal{C B}[p]\right\}$ is a Diophantine subset of the $n_{12}$-xtuples of $\mathbb{N}$. Define $\mathcal{C C}$ [inite 0 -sequence of $\mathbb{N}] \equiv \mathcal{C B}\left[\$_{1}\right]$ and $\mathcal{P C}\left[\$_{1}\right]$. \{ $p$, where $p$ is an $n_{12}$-element finite 0 -sequence of $\mathbb{N}: \mathcal{C C}[p]\}$ is a Diophantine subset of the $n_{12}$-xtuples of $\mathbb{N}$. Define $\mathcal{C D}$ [finite 0 -sequence of $\mathbb{N}] \equiv \mathcal{C C}\left[\$_{1}\right]$ and $\mathcal{P D}\left[\$_{1}\right] .\left\{p\right.$, where $p$ is an $n_{12}$-element finite 0 -sequence of $\mathbb{N}: \mathcal{C D}[p]\}$ is a Diophantine subset of the $n_{12}$-xtuples of $\mathbb{N}$. Define $\mathcal{C E}$ [finite 0 -sequence of $\mathbb{N}] \equiv \mathcal{C D}\left[\$_{1}\right]$ and $\mathcal{P E}\left[\$_{1}\right]$. $\{p$, where $p$ is an $n_{12}$-element finite 0 -sequence of $\left.\mathbb{N}: \mathcal{C E}[p]\right\}$ is a Diophantine subset of the $n_{12}$-xtuples of $\mathbb{N}$. Define $\mathcal{C F}$ [finite 0 -sequence of $\left.\mathbb{N}\right] \equiv \mathcal{C E}\left[\$_{1}\right]$ and $\mathcal{P F}\left[\$_{1}\right]$. Set $P P=\left\{p\right.$, where $p$ is an $n_{12}$-element finite 0 -sequence of $\mathbb{N}$ : $\mathcal{C F}[p]\} . P P$ is a Diophantine subset of the $n_{12}$-xtuples of $\mathbb{N}$. Reconsider $P P_{n}=\left\{p\left\lceil n\right.\right.$, where $p$ is an $n_{12}$-element finite 0 -sequence of $\left.\mathbb{N}: p \in P P\right\}$ as a Diophantine subset of the $n$-xtuples of $\mathbb{N}$. $P P_{n} \subseteq R R . R R \subseteq P P_{n}$.

(35) $\quad\left\{p: p\left(i_{3}\right)=\prod\left(1+p\left(i_{1}\right) \cdot\left(\operatorname{idseq}\left(p\left(i_{2}\right)\right)\right)\right)\right.$ and $\left.p\left(i_{1}\right) \geqslant 1\right\}$ is a Diophantine subset of the $n$-xtuples of $\mathbb{N}$. The theorem is a consequence of (34).

(36) $\quad\left\{p: p\left(i_{3}\right)=\prod\left(1+p\left(i_{1}\right) ! \cdot\left(\operatorname{idseq}\left(1+p\left(i_{2}\right)\right)\right)\right)\right\}$ is a Diophantine subset of the $n$-xtuples of $\mathbb{N}$.

Proof: Define $\mathcal{R}$ (natural number, natural number, natural number) $=\$ 1$ !. For every $i_{1}, i_{2}, i_{3}$, and $i_{4},\left\{p: \mathcal{R}\left(p\left(i_{1}\right), p\left(i_{2}\right), p\left(i_{3}\right)\right)=p\left(i_{4}\right)\right\}$ is a Diophantine subset of the $n$-xtuples of $\mathbb{N}$. Define $\mathcal{P}_{1}$ [natural number, natural number, natural object, natural number, natural number, natural number] $\equiv \$_{1}=\prod\left(1+\$_{3} \cdot\left(\operatorname{idseq}\left(\$_{2}\right)\right)\right)$ and $\$_{3} \geqslant 1$. For every $i_{1}, i_{2}, i_{3}, i_{4}, i_{5}$, and $i_{6},\left\{p: \mathcal{P}_{1}\left[p\left(i_{1}\right), p\left(i_{2}\right), p\left(i_{3}\right), p\left(i_{4}\right), p\left(i_{5}\right), p\left(i_{6}\right)\right]\right\}$ is a Diophantine subset of the $n$-xtuples of $\mathbb{N}$.

For every $i_{1}, i_{2}, i_{3}, i_{4}$, and $i_{5},\left\{p: \mathcal{P}_{1}\left[p\left(i_{1}\right), p\left(i_{2}\right), \mathcal{R}\left(p\left(i_{3}\right), p\left(i_{4}\right), p\left(i_{5}\right)\right)\right.\right.$, $\left.\left.p\left(i_{3}\right), p\left(i_{4}\right), p\left(i_{5}\right)\right]\right\}$ is a Diophantine subset of the $n$-xtuples of $\mathbb{N}$. Define $\mathcal{F}_{2}$ (natural number, natural number, natural number) $=1 \cdot \$_{1}+1$. Define $\mathcal{P}_{2}$ [natural number, natural number, natural object, natural number, natural number, natural number $] \equiv \$_{1}=\prod\left(1+\$_{2} ! \cdot\left(\operatorname{idseq}\left(\$_{3}\right)\right)\right)$ and $\$_{2} ! \geqslant 1$. For every $i_{1}, i_{2}, i_{3}, i_{4}$, and $i_{5},\left\{p: \mathcal{P}_{2}\left[p\left(i_{1}\right), p\left(i_{2}\right), \mathcal{F}_{2}\left(p\left(i_{3}\right), p\left(i_{4}\right), p\left(i_{5}\right)\right), p\left(i_{3}\right)\right.\right.$, $\left.\left.p\left(i_{4}\right), p\left(i_{5}\right)\right]\right\}$ is a Diophantine subset of the $n$-xtuples of $\mathbb{N}$. Define $\mathcal{Q}_{1}$ [finite 
0-sequence of $\mathbb{N}] \equiv \mathcal{P}_{2}\left[\$_{1}\left(i_{3}\right), \$_{1}\left(i_{1}\right), 1 \cdot \$_{1}\left(i_{2}\right)+1,1 \cdot \$_{1}\left(i_{3}\right), \$_{1}\left(i_{3}\right), \$_{1}\left(i_{3}\right)\right]$. Define $\mathcal{Q}_{2}[$ finite 0 -sequence of $\mathbb{N}] \equiv \$_{1}\left(i_{3}\right)=\prod\left(1+\$_{1}\left(i_{1}\right)\right.$ ! $\cdot$ (idseq $(1+$ $\left.\left.\left.\$_{1}\left(i_{2}\right)\right)\right)\right) .\left\{p: \mathcal{Q}_{1}[p]\right\}=\left\{q: \mathcal{Q}_{2}[q]\right\}$.

Let us consider $i_{1}, i_{2}$, and $i_{3}$. Now we state the propositions:

(37) If $n \neq 0$, then $\left\{p: p\left(i_{3}\right)=\prod\left(p\left(i_{2}\right)+1+-\operatorname{idseq}\left(p\left(i_{1}\right)\right)\right)\right.$ and $\left.p\left(i_{2}\right)>p\left(i_{1}\right)\right\}$ is a Diophantine subset of the $n$-xtuples of $\mathbb{N}$.

Proof: Set $n_{2}=n+2$. Define $\mathcal{R}[$ finite 0 -sequence of $\mathbb{N}] \equiv \$_{1}\left(i_{3}\right)=$ $\prod\left(\$_{1}\left(i_{2}\right)+1+-\operatorname{idseq}\left(\$_{1}\left(i_{1}\right)\right)\right)$ and $\$_{1}\left(i_{2}\right)>\$_{1}\left(i_{1}\right)$. Set $R R=\{p: \mathcal{R}[p]\}$. Reconsider $Y=i_{3}, X_{2}=i_{2}, X_{1}=i_{1}, C=n, F=n+1$ as an element of $n_{2}$. Define $\mathcal{P}_{1}$ [finite 0 -sequence of $\left.\mathbb{N}\right] \equiv \$_{1}\left(X_{2}\right) \geqslant \$_{1}\left(X_{1}\right)$ and $\$_{1}(C)=$ $\left(\begin{array}{l}\$_{1}\left(X_{2}\right) \\ \$_{1}\left(X_{1}\right)\end{array}\right) .\left\{p\right.$, where $p$ is an $n_{2}$-element finite 0 -sequence of $\left.\mathbb{N}: \mathcal{P}_{1}[p]\right\}$ is a Diophantine subset of the $n_{2}$-xtuples of $\mathbb{N}$. Define $\mathcal{P}_{2}$ [finite 0 -sequence of $\mathbb{N}] \equiv \$_{1}(F)=\$_{1}\left(X_{1}\right)$ !. $\left\{p\right.$, where $p$ is an $n_{2}$-element finite 0 -sequence of $\left.\mathbb{N}: \mathcal{P}_{2}[p]\right\}$ is a Diophantine subset of the $n_{2}$-xtuples of $\mathbb{N}$. Define $\mathcal{P}_{3}$ [finite 0-sequence of $\mathbb{N}] \equiv 1 \cdot \$_{1}\left(X_{2}\right)>1 \cdot \$_{1}\left(X_{1}\right)+0$. Define $\mathcal{P}_{4}$ [finite 0 -sequence of $\mathbb{N}] \equiv 1 \cdot \$_{1}(Y)=1 \cdot \$_{1}(F) \cdot \$_{1}(C)$.

Define $\mathcal{P}_{12}[$ finite 0 -sequence of $\mathbb{N}] \equiv \mathcal{P}_{1}\left[\$_{1}\right]$ and $\mathcal{P}_{2}\left[\$_{1}\right] .\{p$, where $p$ is an $n_{2}$-element finite 0 -sequence of $\left.\mathbb{N}: \mathcal{P}_{12}[p]\right\}$ is a Diophantine subset of the $n_{2}$-xtuples of $\mathbb{N}$. Define $\mathcal{P}_{123}$ [finite 0 -sequence of $\left.\mathbb{N}\right] \equiv \mathcal{P}_{12}\left[\$_{1}\right]$ and $\mathcal{P}_{3}\left[\$_{1}\right]$. $\left\{p\right.$, where $p$ is an $n_{2}$-element finite 0 -sequence of $\left.\mathbb{N}: \mathcal{P}_{123}[p]\right\}$ is a Diophantine subset of the $n_{2}$-xtuples of $\mathbb{N}$. Define $\mathcal{P}_{1234}$ [finite 0 -sequence of $\mathbb{N}] \equiv \mathcal{P}_{123}\left[\$_{1}\right]$ and $\mathcal{P}_{4}\left[\$_{1}\right]$. Set $P P=\left\{p\right.$, where $p$ is an $n_{2}$-element finite 0 -sequence of $\left.\mathbb{N}: \mathcal{P}_{1234}[p]\right\} . P P$ is a Diophantine subset of the $n_{2}$-xtuples of $\mathbb{N}$. Reconsider $P P_{n}=\left\{p\left\lceil n\right.\right.$, where $p$ is an $n_{2}$-element finite 0 -sequence of $\mathbb{N}: p \in P P\}$ as a Diophantine subset of the $n$-xtuples of $\mathbb{N}$. $P P_{n} \subseteq R R$. $R R \subseteq P P_{n}$

(38) $\left\{p: p\left(i_{3}\right)=\prod\left(p\left(i_{2}\right)+1+-\operatorname{idseq}\left(p\left(i_{1}\right)\right)\right)\right.$ and $\left.p\left(i_{2}\right)>p\left(i_{1}\right)\right\}$ is a Diophantine subset of the $n$-xtuples of $\mathbb{N}$. The theorem is a consequence of (37).

(39) $\left\{p: p\left(i_{1}\right)=\prod\left(i+p_{\left\lfloor n_{1}\right.}\left\lceil n_{2}\right)\right\}\right.$ is a Diophantine subset of the $n$-xtuples of $\mathbb{N}$.

Proof: Define $\mathcal{P}$ [natural number] $\equiv$ for every $n$ such that $\$_{1}+n_{1} \leqslant n$ for every $i_{1},\left\{p: p\left(i_{1}\right)=\prod\left(i+p_{\left\lfloor n_{1}\right.}\left\lceil \$_{1}\right)\right\}\right.$ is a Diophantine subset of the $n$ xtuples of $\mathbb{N}$. $\mathcal{P}[0]$. If $\mathcal{P}[m]$, then $\mathcal{P}[m+1] . \mathcal{P}[m]$.

\section{REFERENCES}

[1] Marcin Acewicz and Karol Pak. Basic Diophantine relations. Formalized Mathematics, 26(2):175-181, 2018. doi 10.2478/forma-2018-0015.

[2] Zofia Adamowicz and Paweł Zbierski. Logic of Mathematics: A Modern Course of Classical 
Logic. Pure and Applied Mathematics: A Wiley Series of Texts, Monographs and Tracts. Wiley-Interscience, 1997.

[3] Grzegorz Bancerek, Czesław Byliński, Adam Grabowski, Artur Korniłowicz, Roman Matuszewski, Adam Naumowicz, Karol Pąk, and Josef Urban. Mizar: State-of-the-art and beyond. In Manfred Kerber, Jacques Carette, Cezary Kaliszyk, Florian Rabe, and Volker Sorge, editors, Intelligent Computer Mathematics, volume 9150 of Lecture Notes in Computer Science, pages 261-279. Springer International Publishing, 2015. ISBN 978-3319-20614-1. doi 10.1007/978-3-319-20615-8_17.

[4] Grzegorz Bancerek, Czesław Byliński, Adam Grabowski, Artur Korniłowicz, Roman Matuszewski, Adam Naumowicz, and Karol Pak. The role of the Mizar Mathematical Library for interactive proof development in Mizar. Journal of Automated Reasoning, 61(1):9-32, 2018. do1:10.1007/s10817-017-9440-6

[5] Martin Davis. Hilbert's tenth problem is unsolvable. The American Mathematical Monthly, Mathematical Association of America, 80(3):233-269, 1973. doi:10.2307/2318447.

[6] Adam Grabowski, Artur Korniłowicz, and Adam Naumowicz. Four decades of Mizar. Journal of Automated Reasoning, 55(3):191-198, 2015. doi 10.1007/s10817-015-9345-1

[7] Artur Korniłowicz and Karol Pak. Basel problem - preliminaries. Formalized Mathematics, 25(2):141-147, 2017. doi 10.1515/forma-2017-0013

[8] Xiquan Liang, Li Yan, and Junjie Zhao. Linear congruence relation and complete residue systems. Formalized Mathematics, 15(4):181-187, 2007. doi 10.2478/v10037-007-0022-7.

[9] Karol Pak. Diophantine sets. Preliminaries. Formalized Mathematics, 26(1):81-90, 2018. doi $10.2478 /$ forma-2018-0007

[10] Craig Alan Smorynski. Logical Number Theory I, An Introduction. Universitext. SpringerVerlag Berlin Heidelberg, 1991. ISBN 978-3-642-75462-3.

[11] Tetsuya Tsunetou, Grzegorz Bancerek, and Yatsuka Nakamura. Zero-based finite sequences Formalized Mathematics, 9(4):825-829, 2001.

[12] Rafał Ziobro. On subnomials. Formalized Mathematics, 24(4):261-273, 2016. doi: $10.1515 /$ forma-2016-0022.

Accepted May 27, 2019 